\title{
Clinicopathological Evaluation and Prognostic Analysis in Breast Cancer Patients with Brain Metastasis
}

\author{
Naoko Minatani*, Hirokazu Tanino, Yoshimasa Kosaka, Mariko Kikuchi, Hiroshi Nishimiya, \\ Mina Waraya, Hiroshi Katoh, Takumo Enomoto, Norihiko Sengoku, Masahiko Watanabe \\ Department of Surgery, Kitasato University School of Medicine, Kanagawa, Japan \\ Email: "minaoko@kitasato-u.ac.jp
}

Received 9 July 2015; accepted 7 August 2015; published 10 August 2015

Copyright (C) 2015 by authors and Scientific Research Publishing Inc.

This work is licensed under the Creative Commons Attribution International License (CC BY). http://creativecommons.org/licenses/by/4.0/

c) (i) Open Access

\begin{abstract}
Background: Brain metastases are diagnosed in 5\% - 15\% of patients with breast cancer. Clinical management of brain metastases appears to be crucial in improving the prognosis of patients with breast cancer that has metastasized to the brain. However, few studies have investigated whether treatment of metastatic brain tumors would lead to improved prognosis. Methods: A total of 41 breast cancer patients whose tumors metastasized to the brain and who were diagnosed and treated at our hospital were enrolled. This study was conducted to determine the characteristics of brain metastases and to identify the factors that may affect the treatment and prognosis of patients with brain metastases. Results: There were more patients with HER2 and triple negative subtypes than of the luminal subtype. The median time from the initiation of breast cancer treatment to the onset of brain metastasis was 31 months (range, 7 - 134 months). Significantly greater disease-specific survival (DSS) was observed in patients who had a performance status of 0 - 2 at the time of diagnosis of metastatic brain tumors than those with a performance status of $3-4(p=$ 0.04). DSS was also significantly greater in patients who underwent surgery to remove brain metastases and in patients treated with systemic therapy ( $p=0.0007$ and 0.0001 , respectively). Conclusions: It may be possible to improve the prognosis of patients with brain metastases if lesions are detected early enough when the patients' performance status is still good. In order for these findings to be definitive, however, results of future prospective studies are eagerly awaited.
\end{abstract}

\section{Keywords}

Brain Metastasis, Breast Cancer, Performance Status

\footnotetext{
${ }^{*}$ Corresponding author.
}

How to cite this paper: Minatani, N., Tanino, H., Kosaka, Y., Kikuchi, M., Nishimiya, H., Waraya, M., Katoh, H., Enomoto, T., Sengoku, N. and Watanabe, M. (2015) Clinicopathological Evaluation and Prognostic Analysis in Breast Cancer Patients with Brain Metastasis. Journal of Cancer Therapy, 6, 727-734. http://dx.doi.org/10.4236/jct.2015.68079 


\section{Introduction}

In Japan, the incidence of cancer in women has been increasing every year. After adjusting for age, breast cancer ranks as the most common cause of cancer death among women. Despite advances in pharmacotherapy for metastatic breast cancer, the number of drugs effective in treating brain metastasis, which are seen in $5 \%-15 \%$ of breast cancer case [1], is limited. The median survival time is three to six months from the time of diagnosis of the brain metastasis, rendering the prognosis poor [2]-[4]. Clinical management of brain metastasis appears to be crucial in improving the prognosis of patients with breast cancer that has metastasized to the brain. However, very little is known about brain metastasis, and few studies have investigated whether treatment of metastatic brain tumors leads to improved prognosis [5] [6].

In this study, we evaluated patients who were treated at our hospital in order to determine the characteristics of brain metastasis and to identify factors that may affect the prognosis of patients with brain metastasis.

\section{Patients and Methods}

A total of 41 breast cancer patients who were diagnosed with metastatic brain tumors at our hospital between January 2000 and November 2012 were enrolled. We sought to identify a possible relationship between characteristics of the patients (shown in Table 1) and brain metastasis-free survival (BMFS) and disease-specific survival (DSS).

\section{Statistical Analysis}

Statistical analysis was performed using the JMP 10 software (SAS Institute Japan Ltd.). The chi-square test and the log-rank test were used for the analysis. A $p<0.05$ was considered significant.

\section{Results}

\subsection{Patient Characteristics}

Patient characteristics are presented in Table 1 . The median age of the patients was 58 years (range, 30 - 82 years). Histological examination revealed that 32 patients had ductal carcinoma, five had lobular carcinoma, two had mucinous adenocarcinoma, and two had tumors of unknown type. Patients with ductal carcinoma were further classified according to nuclear grade. Grade III tumors were found most frequently; 2 patients (6.3\%), 9 patients (28.1\%) and 21 patients (65.6\%) were of grade I, II, and III, respectively. Patients were also classified according to breast cancer subtype: 15 patients (36.6\%) were classified as having estrogen receptor (ER)-negative/ human epidermal growth factor receptor 2 (HER2)-negative tumors; 13 patients (31.7\%) were classified as having ER-/HER2 + tumors; 10 patients (24.4\%) were classified as having ER+/HER2 - tumors; and three patients (7.3\%) were classified as having ER+/HER2+ tumors. At the time that breast cancer treatment was initiated, 18 patients were stage II, 14 patients were stage III, and nine patients were stage IV.

The median recurrence-free survival (RFS) time, which is defined as the time period between the initiation of breast cancer treatment to the development of distant metastasis, was 13 months (range, 1 - 67 months). The median time from the start of breast cancer treatment to the onset of metastatic brain disease was 31 months (range, 7 - 134 months). 12 patients had a solitary metastatic tumor in the brain while 29 patients had more than one metastatic tumor. When brain metastasis were found, eight patients had an Eastern Cooperative Oncology Group Performance Status (ECOG PS) of 0, 10 patients had an ECOG PS of 1, nine patients had an ECOG PS of 2, eight patients had an ECOG PS of 3, and six patients had an ECOG PS of 4. Due to brain metastasis, the ECOG PS deteriorated in 32 out of 33 patients.

\subsection{Cause of Death}

The median observation period was 46 months (range, 6 - 154 months). Of the 41 patients, three patients were still alive at the end of the observation period, while 38 patients died during that time period. 30 patients died from brain metastasis, six patients died from metastatic disease to organs other than the brain, and two patients died of other causes (suicide and drowning at home). Comparison of patients who died from brain metastasis with those who died from metastatic disease to other organs revealed no significant differences in parameters 
Table 1. Clinicopathologic characteristics of the patients.

\begin{tabular}{|c|c|c|}
\hline Factors & No. & $\%$ \\
\hline Patients & 41 & 100.0 \\
\hline Age (mean) & \multicolumn{2}{|c|}{$57.5(30.8-82.3)$} \\
\hline \multicolumn{3}{|l|}{ Pathological type } \\
\hline Ductal carcinoma & 32 & 78.0 \\
\hline Lobular carcinoma & 5 & 12.2 \\
\hline Mucinos carcinoma & 2 & 4.9 \\
\hline Unknown & 2 & 4.9 \\
\hline \multicolumn{3}{|l|}{ Nuclear grade } \\
\hline 1 & 2 & 6.3 \\
\hline 2 & 9 & 28.1 \\
\hline 3 & 21 & 65.6 \\
\hline \multicolumn{3}{|l|}{ Subtype } \\
\hline ER+/HER2+ & 3 & 7.3 \\
\hline ER+/HER2- & 10 & 24.4 \\
\hline ER-/HER2+ & 13 & 31.7 \\
\hline ER-/HER2- & 15 & 36.6 \\
\hline \multicolumn{3}{|l|}{ Tumor size } \\
\hline $\mathrm{T} 1$ & 4 & 9.8 \\
\hline $\mathrm{T} 2$ & 25 & 61.0 \\
\hline T3 & 4 & 9.8 \\
\hline $\mathrm{T} 4$ & 8 & 19.4 \\
\hline \multicolumn{3}{|l|}{ Lymph node metastasis } \\
\hline Negative & 11 & 26.8 \\
\hline Positive & 30 & 73.2 \\
\hline \multicolumn{3}{|l|}{ Stage $\left({ }^{*} 1\right)$} \\
\hline 2 & 18 & 43.9 \\
\hline 3 & 14 & 34.1 \\
\hline 4 & 9 & 22.0 \\
\hline \multicolumn{3}{|l|}{ Number of brain metastasis } \\
\hline 1 & 12 & 29.3 \\
\hline $1<$ & 29 & 70.7 \\
\hline \multicolumn{3}{|l|}{ ECOG PS } \\
\hline 0 & 8 & 19.5 \\
\hline 1 & 10 & 24.4 \\
\hline 2 & 9 & 22.0 \\
\hline 3 & 8 & 19.5 \\
\hline 4 & 6 & 14.6 \\
\hline
\end{tabular}

ER: Estrogen receptor; ECOG PS: Eastern Cooperative Oncology Group Performance Status; HER2 negative: 0 - 2, positive: 3. " 1 : 7th edition of the Union for International Cancer Control (UICC). 
such as age, histological classification, subtype, stage, the number of brain metastasis, and ECOG PS score (Table 2). Of those patients who underwent brain surgery to remove the metastatic tumor, two patients died due to metastasis to other organs.

\subsection{Treatment of Brain Metastasis}

The treatment of brain metastasis in patients with breast cancer was as follows: whole-brain radiation therapy in 35 patients; tumorectomy for brain metastasis in four patients, three of those patients received both treatments; and systemic therapy in 19 patients (Table 2).

\subsection{Analysis of Clinical Prognosis}

Of the 41 patients with metastatic brain cancer, those with negative lymph node metastasis had better brain metastasis-free survival (BMFS) than those with positive lymph node metastasis (Figure 1). Patients who had an ECOG PS of 0 - 2 when the metastatic brain tumor was found had greater disease-specific survival (DSS) than those whose scores were 3 - 4. Patients who underwent tumorectomy for brain metastasis and those who were treated with chemotherapy had significantly prolonged DSS (Figure 2). There was no significant difference in

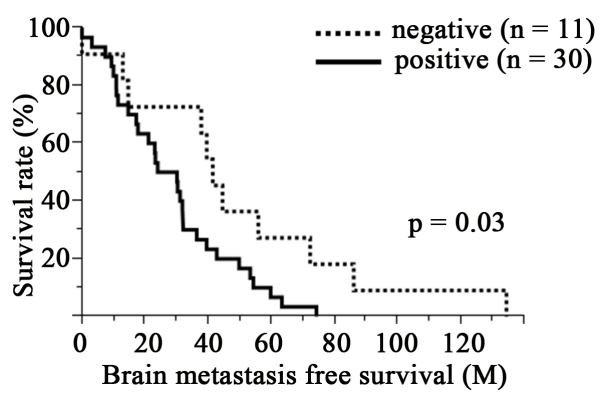

Figure 1. Patients with negative lymph node metastasis had longer BMFS than those with positive lymph node metastases.
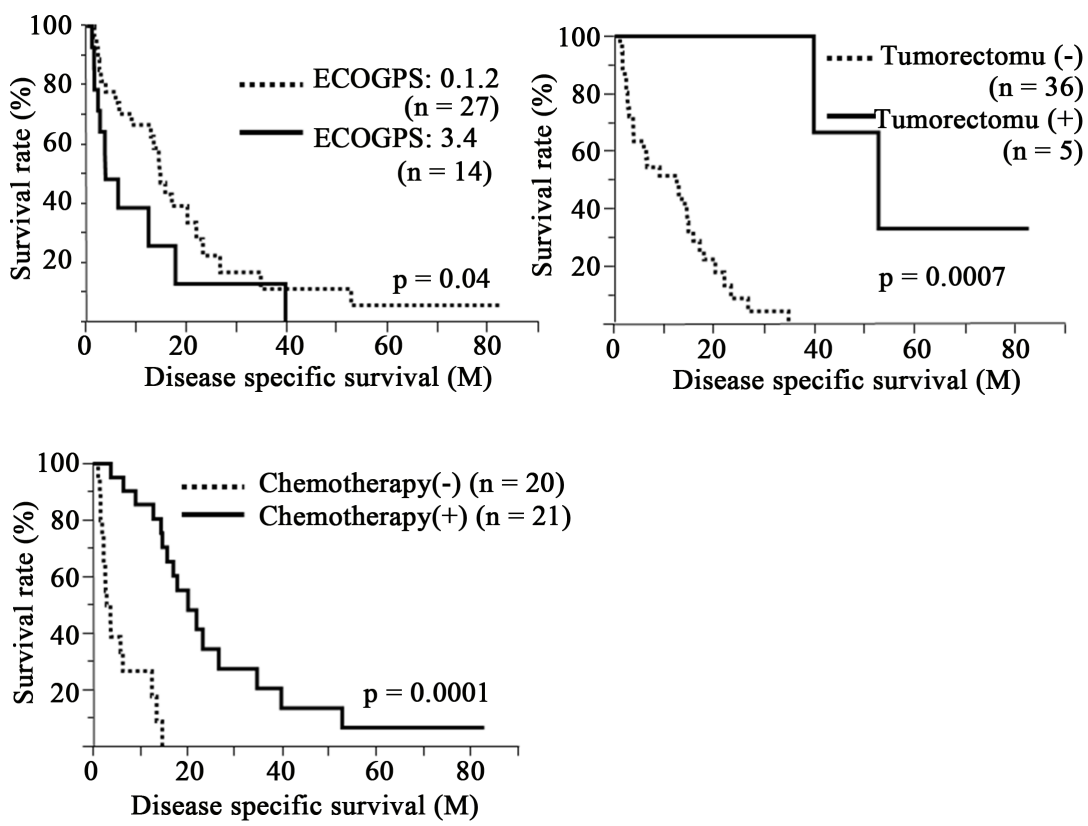

Figure 2. Factors associated with DSS. Disease-specific survival (DSS) was observed in patients who had a performance status of 0 - 2 at the time of diagnosis of metastatic brain tumors than those with a performance status of 3 - $4(p=0.04)$. DSS was also significantly greater in patients who underwent surgery to remove brain metastases and in patients treated with systemic therapy. 
Table 2. Correlation of clinicopathologic characteristics and cause of death.

\begin{tabular}{|c|c|c|c|c|c|c|}
\hline \multirow{4}{*}{ Factors } & \multirow{4}{*}{$\mathrm{n}$} & \multicolumn{4}{|c|}{ Cause of death } & \multirow{4}{*}{$p$} \\
\hline & & \multirow{2}{*}{\multicolumn{2}{|c|}{$\begin{array}{l}\text { Brain metastasis } \\
\qquad(\mathrm{n}=30)\end{array}$}} & \multirow{2}{*}{\multicolumn{2}{|c|}{$\begin{array}{l}\text { Other sites metstasis } \\
\qquad(\mathrm{n}=5)\end{array}$}} & \\
\hline & & & & & & \\
\hline & & No. & $\%$ & No. & $\%$ & \\
\hline Age (mean) & & \multicolumn{2}{|c|}{$56.9(30.8-82.3)$} & \multicolumn{2}{|c|}{$57.1(49.7$ - 63.6) } & 0.96 \\
\hline \multicolumn{7}{|l|}{ Pathological type } \\
\hline Ductal carcinoma & 28 & 23 & 76.7 & 5 & 100.0 & 0.11 \\
\hline Other & 7 & 7 & 23.3 & 0 & 0.0 & \\
\hline \multicolumn{7}{|l|}{ Nuclear grade } \\
\hline $1 / 2$ & 19 & 15 & 65.2 & 4 & 80.0 & 0.50 \\
\hline 3 & 9 & 8 & 34.8 & 1 & 20.0 & \\
\hline \multicolumn{7}{|l|}{ Estrogen receptor } \\
\hline Negative & 25 & 23 & 76.7 & 2 & 40.0 & 0.06 \\
\hline Positive & 10 & 7 & 23.3 & 3 & 60.0 & \\
\hline \multicolumn{7}{|l|}{ HER-2/NEU } \\
\hline Negative & 14 & 12 & 40.0 & 2 & 30.0 & 1.00 \\
\hline Positive & 21 & 18 & 60.0 & 3 & 70.0 & \\
\hline \multicolumn{7}{|l|}{ Tumor size } \\
\hline $\mathrm{T} 1$ & 4 & 3 & 9.7 & 1 & 10.0 & 0.54 \\
\hline $\mathrm{T} 2-4$ & 31 & 27 & 90.3 & 4 & 90.0 & \\
\hline \multicolumn{7}{|l|}{ Lymph node metastasis } \\
\hline Negative & 10 & 9 & 10.0 & 1 & 20.0 & 0.64 \\
\hline Positive & 25 & 21 & 90.0 & 4 & 80.0 & \\
\hline \multicolumn{7}{|l|}{ Stage } \\
\hline 2 & 17 & 15 & 50.0 & 2 & 40.0 & 0.23 \\
\hline 3 & 12 & 9 & 30.0 & 3 & 60.0 & \\
\hline 4 & 6 & 6 & 20.0 & 0 & 0.0 & \\
\hline \multicolumn{7}{|c|}{ Number of brain metastasis } \\
\hline 1 & 9 & 7 & 23.3 & 2 & 40.0 & 0.44 \\
\hline $1<$ & 26 & 23 & 76.7 & 3 & 60.0 & \\
\hline \multicolumn{7}{|l|}{ ECOG PS } \\
\hline $0-1$ & 16 & 14 & 46.7 & 2 & 40.0 & 0.78 \\
\hline $2-4$ & 19 & 16 & 53.3 & 3 & 60.0 & \\
\hline \multicolumn{7}{|c|}{ Therapy of brain metastasis } \\
\hline \multicolumn{7}{|c|}{ Whole brain radiotherapy } \\
\hline+ & 31 & 27 & 90.0 & 4 & 80.0 & 0.54 \\
\hline- & 4 & 3 & 30.0 & 1 & 20.0 & \\
\hline \multicolumn{7}{|l|}{ Tumorectomy } \\
\hline+ & 2 & 0 & 0.0 & 2 & 40.0 & 0.003 \\
\hline- & 33 & 30 & 100.0 & 3 & 60.0 & \\
\hline \multicolumn{7}{|l|}{ Systemic therapy } \\
\hline+ & 16 & 13 & 43.3 & 3 & 60.0 & 0.49 \\
\hline- & 19 & 17 & 56.7 & 2 & 20.0 & \\
\hline
\end{tabular}


BMFS or DSS between patients who died from brain metastasis and patients who died from metastatic disease to organs other than the brain ( $p=0.37$ and 0.91 , respectively). Similarly, there was no significant association between BMFS or DSS and patient characteristics such as the status of estrogen receptors, the status of HER2 receptors, histological differences in tumors, primary breast tumor size, tumor stage, or the number of metastatic brain lesions. In patients with brain metastasis, the time interval from the detection of breast cancer to its metastasis to the brain was not associated with the prognosis (Figure 3).

\section{Discussion}

Among the patients in the current study, the time period between the detection of breast cancer to its metastasis to the brain was not correlated with the clinical prognosis. On the other hand, patients with better ECOG PS scores had more favorable prognoses than those with poorer scores. A previous study [7] has also reported that the overall survival (OS) of patients after brain metastasis depends on ECOG PS scores. We found that the development of metastatic tumors in the brain was associated with the deterioration of performance status in 32 out of 33 patients.

Patients who underwent tumorectomy for brain metastasis and those who were treated with chemotherapy had significantly prolonged DSS. The rate of patients who received pharmacotherapy was greater in those with good performance status than those with poor performance status $(p=0.0004)$. These findings suggest that longer DSS can be achieved if brain metastases are detected early before these tumors begin to affect the patients' performance status, and if the patients are treated with brain tumorectomy and systemic therapy. However, it was difficult to eliminate lead-time bias from our data. Therefore, prospective studies are needed to address whether these treatments truly improves prognosis.

Graesslin et al. have developed a nomogram to identify patients who are at high risk for developing brain metastasis (Figure 4). Using the nomogram, they outline a clinical trial in this subset of patients who are given prophylactic radiation in the hopes of preventing brain metastasis [8]. According to their study, patients with a $45 \%$ probability of developing brain metastasis comprise $20 \%$ of breast cancer patients. Graesslin and colleagues estimate a $60 \%$ reduction in the development of brain metastasis when prophylactic brain irradiation is administered to this select population of patients.

All of the 41 patients who developed brain metastasis at our hospital between 2000 and 2012 had a $\leq 30 \%$ probability of developing brain metastasis as determined by the nomogram. Therefore, our patients were considered low risk according to Graesslin's nomogram. The probability of developing metastatic brain tumors according to the nomogram was different from the actual incidence of brain metastasis in our patients. Whether prophylactic cranial irradiation is beneficial remains unsolved today, underscoring the need for future clinical trials in this area.

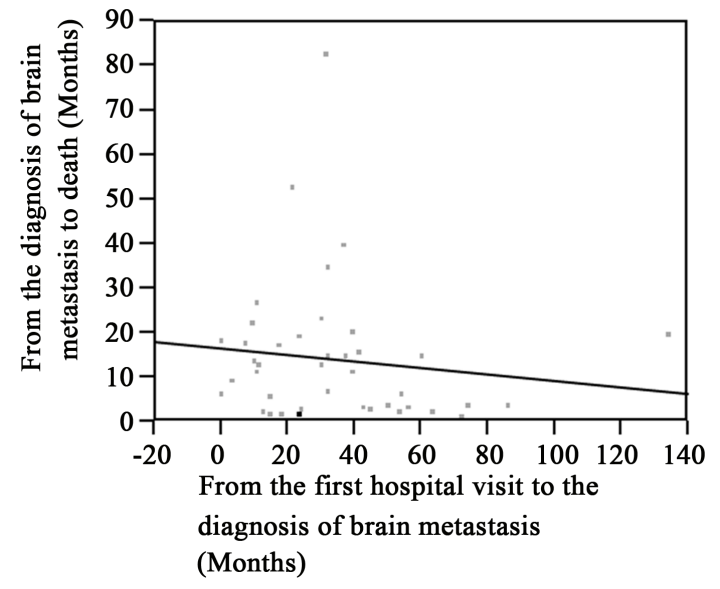

Figure 3. The relationship between the duration from treatment initiation to brain metastasis and the duration from brain metastasis to death. The time interval from the detection of breast cancer to its metastasis to the brain was not associated with the prognosis. 


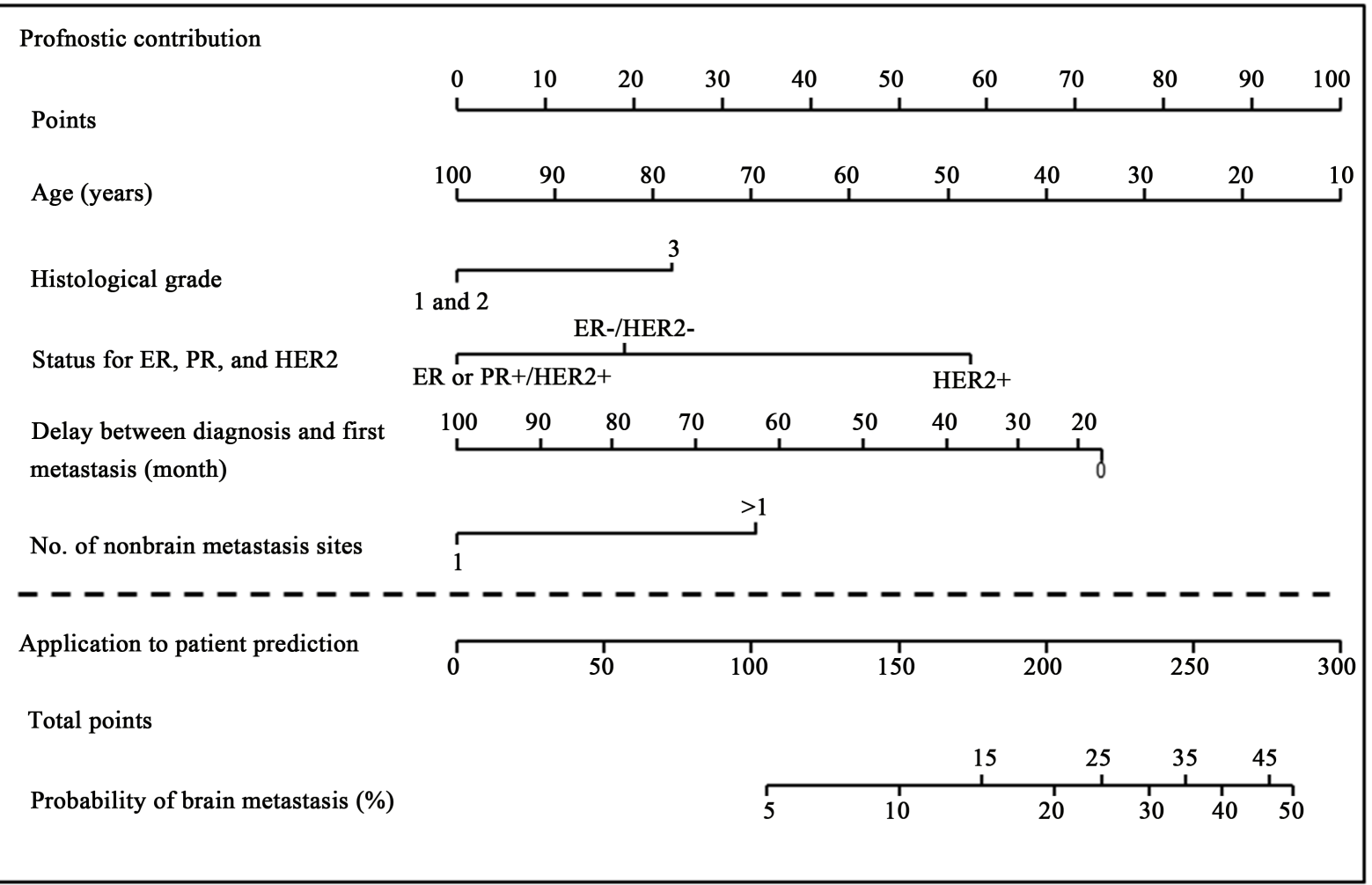

Figure 4. Nomogram to predict the probability of brain metastasis. Nomogram consulted material used in M. D. Anderson Cancer Center.

In Japan, a clinical trial is currently underway in patients who are at high risk of recurrence after radical resection of breast cancer (JCOG 1204). The aim of the study is to determine whether intensive follow-up, including the detection of brain metastasis, will translate into better prognosis such as longer survival. Further clinical data are necessary to evaluate the early detection of brain metastasis for improvement of the prognosis of the metastatic breast cancer patients.

\section{Conflict of Interest}

The authors declare that they have no conflict of interest.

\section{References}

[1] Siegel, R., Ward, E., Brawley, O. and Jemal, A. (2011) Cancer Statistics, 2011: The Impact of Eliminating Socioeconomic and Racial Disparities on Premature Cancer Deaths. CA: A Cancer Journal for Clinicians, 61, 212-236. http://dx.doi.org/10.3322/caac.20121

[2] DiStefano, A., Yong Yap, Y., Hortobagyi, G.N. and Blumenschein, G.R. (1979) The Natural History of Breast Cancer Patients with Brain Metastases. Cancer, 44, 1913-1918. http://dx.doi.org/10.1002/1097-0142(197911)44:5<1913::AID-CNCR2820440554>3.0.CO;2-D

[3] Marko, N.F., Xu, Z., Gao, T., Kattan, M.W. and Weil, R.J. (2011) Predicting Survival in Women with Breast Cancer and Brain Metastasis: A Nomogram Outperforms Current Survival Prediction Models. Cancer, 118, 3749-3757. http://dx.doi.org/10.1002/cncr.26716

[4] Tsukada, Y., Fouad, A., Pickren, J.W. and Lane, W.W. (1983) Central Nervous System Metastasis from Breast Carcinoma. Autopsy Study. Cancer, 52, 2346-2354. http://dx.doi.org/10.1002/1097-0142(19831215)52:12<2349::AID-CNCR2820521231>3.0.CO;2-B

[5] Kaplan, M.A., Ertugrul, H. and Firat, U. (2014) Brain Metastases in HER2-Positive Metastatic Breast Cancer Patients Who Received Chemotherapy with or without Trastuzumab. Breast Cancer.

[6] Lin, N.U., Bellon, J.R. and Winer, E.P. (2004) CNS Metastases in Breast Cancer. Journal of Clinical Oncology, 22, 
3608-3617. http://dx.doi.org/10.1200/JCO.2004.01.175

[7] Tarhan, M.O., Demir, L., Somali, I., Yigit, S., Erten, C., Alacacioglu, A., et al. (2013) The Clinicopathological Evaluation of the Breast Cancer Patients with Brain Metastases: Predictors of Survival. Clinical \& Experimental Metastasis, 30, 201-213. http://dx.doi.org/10.1007/s10585-012-9528-7

[8] Graesslin, O., Abdulkarim, B.S., Coutant, C., Huguet, F., Gabos, Z., Hsu, L., Marpeau, O., et al. (2010) Nomogram to Predict Subsequent Brain Metastasis in Patients with Metastatic Breast Cancer. Journal of Clinical Oncology, 28, 2032-2037. http://dx.doi.org/10.1200/JCO.2009.24.6314 